\title{
Integration of vehicle dynamics control systems with an extendable bumper for collision mitigation
}

\author{
M. Elkady ${ }^{1,2, *}$, A. Elmarakbi ${ }^{3}$ and J. MacIntyre ${ }^{3}$ \\ ${ }^{1}$ Department of Mechanical Engineering, School of Engineering, \\ Lebanese International University (LIU)1, Mouseitbeh - Beirut - Lebanon \\ *E-mail: mostafa.kady@liu.edu.lb \\ ${ }^{2}$ Department of Automotive Engineering, Faculty of Engineering, \\ Ain Shams University, Cairo, Egypt \\ ${ }^{3}$ Department of Computing, Engineering and Technology, University of Sunderland, \\ The Sir Tom Cowie Campus at St Peter's, St Peter's Way, Sunderland, SR6 0DD, UK \\ E-mail: ahmed.elmarakbi@sunderland.ac.uk \\ Fax: +44 (191) 5152781
}

\begin{abstract}
The aim of this paper is to enhance crashworthiness in the case of vehicle-to-barrier full frontal collision using vehicle dynamics control systems integrated with an extendable bumper. The work carried out in this paper includes developing and analysing a new vehicle dynamics/crash mathematical model and a multi-body occupant mathematical model. The first model integrates a vehicle dynamics model with the vehicle's front-end structure to define the vehicle's body crash kinematic parameters. In this model, the antilock braking system (ABS) and the active suspension control system (ASC) are cosimulated, and its associated equations of motion are developed. The second model is used to capture the occupant kinematics during full-frontal collision. The numerical simulations show that in the case of using the extendable bumper, the crash energy absorbed is considerable compared to traditional structure. Therefore, the minimum vehicle crumble zone's deformation is obtained when the ABS alongside under pitch control (UPC) is applied with the extendable bumper. The minimum pitch angle of the vehicle body and acceleration are obtained when the ABS alongside UPC technique is applied without the extendable bumper. The occupant deceleration and the occupant's chest and head rotational acceleration are used as injury criteria. The longitudinal displacement and acceleration of the occupant is extremely decreased when the extendable bumper is used. It is also shown that the VDCS can affect the crash characteristics and the occupant safety positively, whereas the rotations angle and acceleration of the occupant chest and head are significantly reduced.
\end{abstract}

Keywords: active safety; collision mitigation; vehicle dynamics adn control; mathematical modelling; extendable bumper

\section{INTRODUCTION}

Nowadays, vehicle safety requirements and collision mitigation are one of the most important points of research in automotive engineering. The increasing public awareness of safety issues and the increasing legislative requirements have increased the pressure on vehicle manufacturers to improve vehicle crashworthiness. Many different techniques have been studied to investigate the opportunities of vehicle collision mitigation. These 
techniques can be classified as pre- and post-collision. The advance driver assistant systems (ADAS) is one of the most well-known method developed to enhance vehicle systems for better driving and safety. They are intended to help avoiding collisions and accidents by providing technologies and collecting data from the road which alert the drivers, or to avoid collisions by implementing self-control of the vehicle. In the event of unavoidable collision, different actions may be taken to minimise the crash. For instance, the brake assistant system (BAS) [1] and the collision mitigation brake system (CMBS) [2] could be used to activate the braking instantly based on the behaviour characteristics of the driver, and relative position from the most dangerous other objects for the moment. In the platoon control, ADAS is considered in order to make the vehicle behave monotonically in the forward direction without collision [3]. While ADAS has been investigated, developed, and is already used for some modern vehicles, it is still far away from its goal to prevent vehicle collisions.

In terms of the enhancing crash energy absorption and minimising deformation of the vehicle's structure in post-collision, two types of smart front-end structures, namely extendable and fixed, have been proposed and analysed to mitigate vehicle collision and enhance crash behaviour in different crash scenarios [4-8]. The extendable smart frontend structure, which is considered in this paper, consists of two hydraulic cylinders integrated with the front-end longitudinal members of standard vehicles. The hydraulic cylinders can be extended in impending collisions using radar techniques to absorb the impact kinetic energy, proving that smart structure can absorb more crash energy by their damping characteristics. For this smart structure, several mathematical models have been developed while analytical and numerical simulations have been presented [7, 8]. In the same way, an extendable and retractable knee bolster was mathematically presented [9]. This can be extended at the threat of an impending collision and retracted if the threat is suppressed. This system was proposed to be positioned in the lower portion of the instrument panel of a vehicle at knee height to an occupant. Another extendable and retractable bumper (E/R bumper) was presented analytically and experimentally [10]. Also, the E/R bumper extends at impending collision to give an extra gap for absorption of crush energy and retracts when the threat disappears. This system provides a maximum bumper extension of $100 \mathrm{~mm}$, which is suitable for a maximum crash speed of about 60 $\mathrm{km} / \mathrm{hr}$. With regards to the occupant's safety, vehicle body pitch and drop during frontal impact play an important role in driver's neck and head injury [11]. Vehicle body pitch and drop have normally been experienced in frontal crash tests. Chang et al. used a finite element (FE) method to investigate frame deformation upon full-frontal impact, in addition to discussing the cause and counter-measures design regarding vehicle body pitch and drop. It was found that downward bending generated from the geometric offsets of the frame rails in the vertical direction during a crash is the key feature of the pitching of the vehicle body.

Modern motor vehicles are increasingly using vehicle dynamic control systems (VDCS) to replace traditional mechanical systems in order to improve vehicle handling, stability, and comfort. In addition, VDCS play an important role for active safety system for road vehicles, which control the dynamic vehicle motion in emergency situations. Anti-lock brake system (ABS) is used to allow the vehicle to follow the desired steering angle while the intense braking is applied [12]. In addition, the ABS helps reducing the stopping distance of a vehicle compared to the conventional braking system. Model predictive control was used in order to stabilise a vehicle during longitudinal or manoeuvring situations [13]. The active suspension control system (ASC) is used to improve the quality of the vehicle ride and reduce the vertical acceleration $[14,15]$. An 
extensive review of the current literatures showed that a little research exists on the influences of vehicle dynamics on vehicle collisions. The influence of the braking force on vehicle impact dynamics in low-speed rear-end collisions has been studied [16]. It was confirmed that the braking force was not negligible in high-quality simulations of vehicle impact dynamics at low speed. The effect of vehicle braking on the crash and the possibility of using vehicle dynamics control systems to reduce the risk of incompatibility and improve the crash performance in frontal vehicle-to-barrier collision were investigated [17]. They proved that there is a slight improvement of the vehicle deformation once the brakes are applied during the crash. A multi-body vehicle dynamic model using ADAMS software, alongside with a simple crash model was generated in order to study the effects of the implemented control strategy.

In this paper, a unique vehicle crash/dynamics mathematical model is developed. This model is used to investigate the mitigation of the vehicle collision in the case of fullfrontal vehicle-to-barrier crash scenario using VDCS and an extendable bumper. In addition, an occupant model is used to capture the occupant kinematics during the collision.

\section{MATHEMATICAL MODELS}

A vehicle frontal collision can be divided into two main stages; the first one is a primary impact, and the second one is a secondary impact. The primary impact indicates the collision between the front-end structure of the vehicle and an obstacle (Barrier in this paper). The secondary impact is the interaction between the occupant and the restraint system and/or the vehicle interior due to vehicle collisions. In this paper, tow mathematical models are developed. The first one (vehicle dynamics/crash model) is used to capture the vehicle crash characteristics, while the second one (occupant model) is used to predict the occupant's behaviour during the collision.

\section{Vehicle Dynamics/Crash Model}

Vehicle modelling was used to simulate vehicle dynamics [18] or vehicle crashes [6,8]. In this paper, a 4-DoF vehicle dynamics/crash mathematical model is developed to study the effect of vehicle dynamics control systems on vehicle collision mitigation. Full-frontal vehicle-to-barrier crash scenario is considered in this paper. The ABS and the ASC systems are co-simulated with a vehicle dynamic model and integrated with a non-linear front-end structure model. The ideal structure needs to have extendable length when the front-end structure is not capable of absorbing crash energy without violating deceleration pulse requirements. A smart structure is used to meet this ideal requirement. The frontend structure consists of two hydraulic cylinders integrated with the front-end longitudinal members of standard vehicles as shown in Figure 1. The general dimensions of the model are shown in Figure 1, where $\mathrm{l}_{\mathrm{f}}, \mathrm{l}_{\mathrm{r}}, \mathrm{h}, \mathrm{e}_{1}$ and $\mathrm{e}_{2}$ represent the longitudinal distance between the vehicle's CG and front wheels, the longitudinal distance between the $\mathrm{CG}$ and rear wheels, the high of the CG from the ground, the distance between the CG and front-end upper springs and the $\mathrm{CG}$ and front-end lower springs respectively. Figure 2 shows the deformation of the front-end and vehicle pitching at the early stage and at the end of impact for the vehicle.

In this half-car model, the vehicle body is represented by lumping mass $\mathrm{m}$ and it has a translational motion in longitudinal direction ( $\mathrm{x}$-axis), translational motion on vertical direction (z-axis) and pitching motion (around $y$-axis). The front-end structure is represented by two non-linear springs with stiffnesses $\mathrm{k}_{\mathrm{su}}$ and $\mathrm{k}_{\mathrm{sl}}$ for the upper members 
(rails) and the lower members of the vehicle frontal structure, respectively. The upper and lower cross members and the bumper are represented by lumped masses $\mathrm{m}_{\mathrm{cu}}, \mathrm{m}_{\mathrm{cl}}$ and $\mathrm{m}_{\mathrm{b}}$, respectively, and they only have a longitudinal motion in the $\mathrm{x}$ direction. The extendable bumper consists of controlled hydraulic cylinder with damping coefficient $\mathrm{c}_{\mathrm{b}}$ integrated with the front-end longitudinal members as shown in Figure 1. Two spring/damper units are used to represent the conventional vehicle suspension systems. Each unit has a spring stiffness $\mathrm{k}_{\mathrm{s}}$ and a damping coefficient $\mathrm{c}$. The subscripts $\mathrm{f}$ and $\mathrm{r}, \mathrm{u}$ and 1 denote the front and rear wheels, upper and lower longitudinal members, respectively. The ASC system is co-simulated with the conventional suspension system to add or subtract an active force element $u$. The ABS is co-simulated with the mathematical model using a simple wheel model. The unsprung masses are not considered in this model and it is assumed that the vehicle moves on a flat-asphalted road, which means that the vertical movement of the tyres and road vertical forces can be neglected.

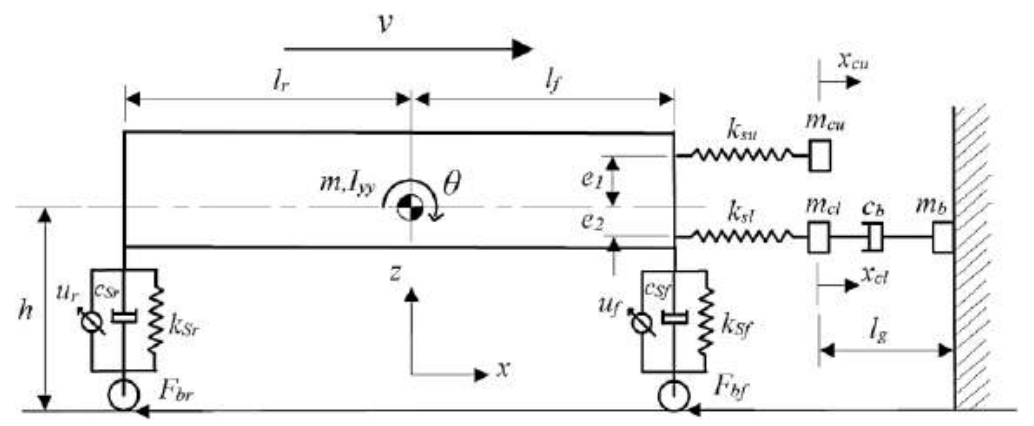

Figure 1. Vehicle crash/dynamics mathematical model.

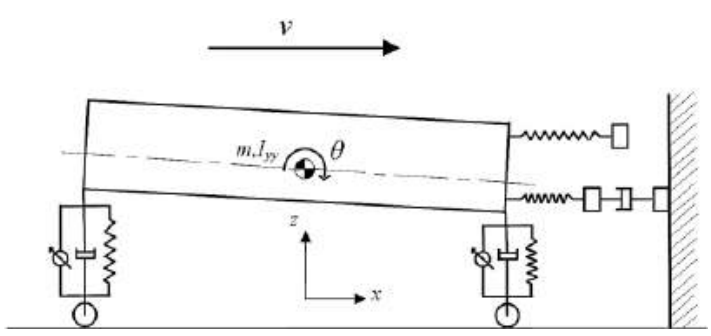

(a)

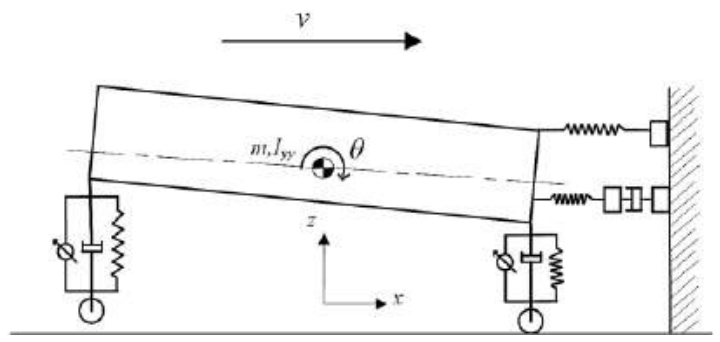

(b)

Figure 2. (a) The mathematical models at the early stage of the impact (b) The mathematical models at the end of impact.

The equations of motion of the mathematical model are developed to study and predict the dynamic response of the vehicle-to-barrier in full frontal crash scenario as follows:

$$
\begin{gathered}
m \cdot \ddot{x}+F_{s u}+F_{s l}+F_{b f}+F_{b r}=0 \\
m \cdot \ddot{z}+F_{S f}+F_{S r}=0 \\
I_{y y} \cdot \ddot{\theta}-F_{S f} \cdot l_{f}+F_{S r} \cdot l_{r}+F_{s u} \cdot d_{1}-F_{s l} \cdot d_{2}-\left(F_{b f}+F_{b r}\right) \cdot(z+h)=0 \\
m_{c l} \cdot \ddot{x}_{c l}+F_{d}-F_{s l}=0
\end{gathered}
$$


At the point of impact, the lumped mass $m_{\text {cu }}$ moves freely until reaching the barrier, then it stops moving until the end of the collision, while the lumped mass $m_{b}$ does not move from the beginning to the end of collision due to the contact with the barrier. Therefore, the two masses $\left(\mathrm{m}_{\mathrm{cu}}\right.$ and $\left.\mathrm{m}_{\mathrm{b}}\right)$ are not included in the equations of motion. The scripts $\ddot{x}$ and $\ddot{z}$ are the acceleration of the vehicle body in longitudinal direction and vertical directions, respectively. $\ddot{\theta}$ is the rotational pitching acceleration of the vehicle body. $F_{s}, F_{S}, F_{b}$ and $F_{d}$ are front-end non-linear spring forces, vehicle suspension forces, braking forces and the damping force of the extendable bumper hydraulic cylinder, respectively. $\ddot{x}_{c l}$ is the longitudinal acceleration of the lower cross member. $\mathrm{d}_{1}$ and $\mathrm{d}_{2}$ represent the distance between the CG and the upper springs force and the lower springs force for each vehicle, respectively and can be calculated using Figure 3 as:

$$
\begin{aligned}
& d_{1}=\sqrt{l_{f}^{2}+e_{1}^{2}} \cdot \sin \left(\tan ^{-1}\left(\frac{e_{1}}{l_{f}}\right)+\theta\right) \\
& d_{2}=\sqrt{l_{f}^{2}+e_{2}^{2}} \cdot \sin \left(\tan ^{-1}\left(\frac{e_{2}}{l_{f}}\right)+\theta\right)
\end{aligned}
$$

There are different types of forces which are applied on the vehicle body. These forces are generated by the deformation of the front-end structure and damping of the extendable bumper due to vehicle crushing, conventional suspension system due to the movement of the vehicle body, and the active control systems such as the ABS and ASC.

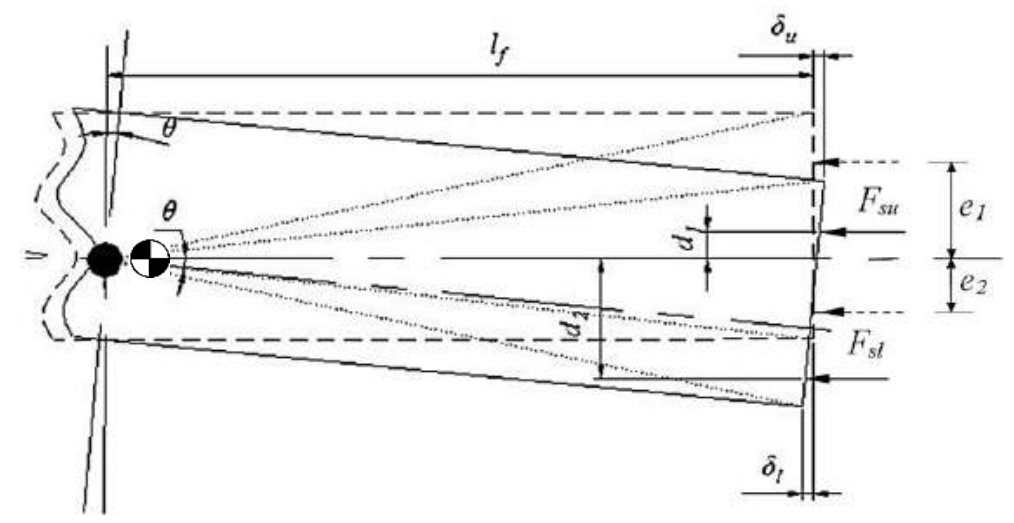

Figure 3. Illustration of the front-end deformation due to vehicle pitching.

$$
\text { ( ----Before pitching — After pitching) }
$$

To simulate the upper and lower members of the vehicle front-end structure, multistage piecewise linear force-deformation spring characteristics are considered. The nonlinear springs used in the multi-body model (ADAMS) [17] are taken to generate the $n$ stage piecewise spring's characteristics. The forces of the front-end springs are calculated using the general relationship between the force and deflection of a non-linear spring depicted in Figure 4 as follows:

$$
F_{s i}=k_{s i j} \delta_{i}+F_{i j}
$$

where $\mathrm{k}_{\mathrm{s}}$ and $\delta$ represent the stiffness and the deflection of the front-end spring, respectively. The subscript $\mathrm{i}$ indicates the spring location (u: upper right spring, l: lower 
right spring) and the subscript $\mathrm{j}$ indicates different stages of the force-deformation characteristics as shown in Figure 4. The stiffness of the spring $\mathrm{k}_{\mathrm{s}}$ and the force elements $\mathrm{F}_{\mathrm{ij}}$ vary according to the different stages of the deflection.

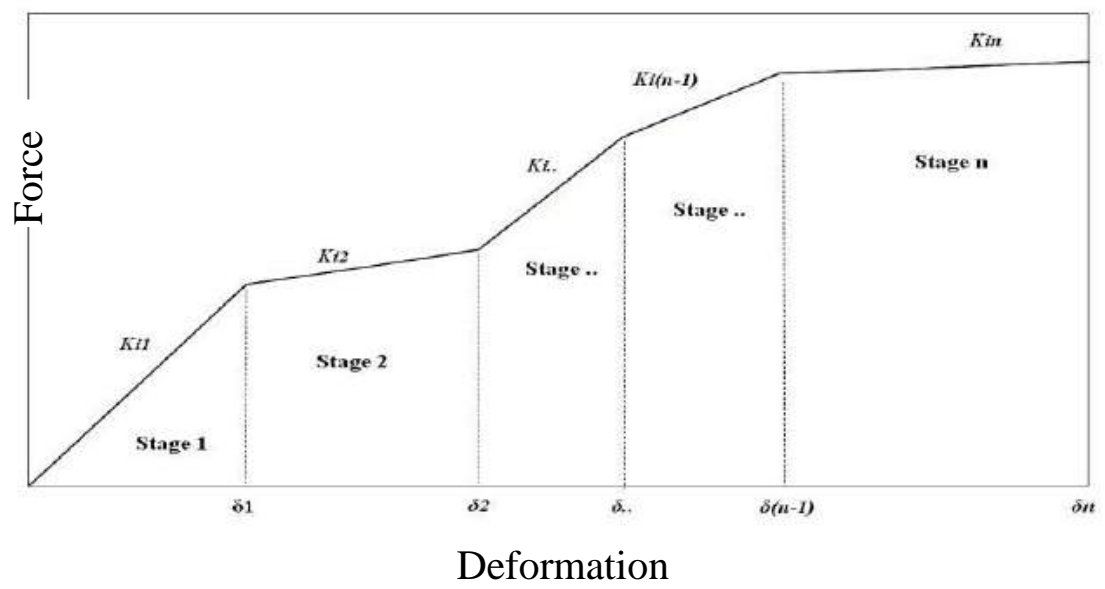

Figure 4. General piecewise force-deformation characteristics.

The damping force of the extendable bumper that is generated from the vehicle crash is expressed as follows:

$$
F_{d}=c_{d} \cdot \dot{x}_{c l}
$$

where $c_{\mathrm{d}}$ is the damping coefficient of the hydraulic cylinder of the extendable bumper, $\dot{x}_{c l}$ is the velocity of the lower cross member. The suspension forces are generated via vertical and pitching movements and the velocity of the vehicle body and can be written as follows:

$$
\begin{gathered}
F_{S f}=k_{S f} \cdot\left(z-l_{f} \cdot \sin \theta\right)+c_{f}\left(\dot{z}-l_{f} \cdot \dot{\theta} \cdot \cos \theta\right)-u_{f} \\
F_{S r}=k_{S r}\left(z+l_{r} \cdot \sin \theta\right)+c_{r}\left(\dot{z}+l_{r} \cdot \dot{\theta} \cdot \cos \theta\right)-u_{r}
\end{gathered}
$$

where $\mathrm{z}$ and $\theta$ are the vehicle body vertical displacement and pitching angle, respectively, and $\dot{z}$ and $\dot{\theta}$ are the vehicle body vertical and pitching velocities, respectively. The ASC force elements $(\mathrm{u})$ are applied in the vertical direction parallel to the existing conventional suspension system.

The ASC force elements are taken in parallel with the existing conventional suspension system and applied in the vertical direction. The maximum active suspension force is considered to be $2000 \mathrm{~N}$ on each wheel with the maximum suspension travel limit of $100 \mathrm{~mm}$, taking into consideration the response time of the ASC system. Validation of the vehicle dynamics-crash model was established in a previous study by the authors to ensure the validity of the model [19]. This is accomplished by comparing the mathematical model results with real test data and the results of the former ADAMS model. The validation test showed that the mathematical model results are well-matched with the other results. 


\section{Multi-Body Occupant Model}

The occupant mathematical model shown in Figure 5 is developed to evaluate the occupant's kinematic behaviour in full frontal crash scenarios. The human body model consists of three bodies, with masses $\mathrm{m}_{1}, \mathrm{~m}_{2}$ and $\mathrm{m}_{3}$ [20]. The first body (lower body), with mass $\mathrm{m}_{1}$, represents the legs and the pelvic area of the occupant and is considered to have a translation motion in the longitudinal direction and rotation motion around the CG of the vehicle. The second body (middle body), with mass $\mathrm{m}_{2}$, represents the occupant's abdominal area, the thorax area and the arms, and is considered to have a translation motion in the longitudinal direction and rotation motion around the pivot between the lower and middle bodies (pivot 1). The third body (upper body), with mass $\mathrm{m}_{3}$, represents the head and neck of the occupant and is considered to have a translation motion in the longitudinal direction and rotation motion around the pivot between the middle and upper bodies (pivot 2). One rotational spring is considered at each pivot to represent the joint stiffness between the pelvic area and the abdominal area and between the thorax area and the neck/head area, respectively. The seatbelt is represented by two linear spring-damper units between the compartment and the occupant; and the airbag is represented by one linear spring-damper unit.
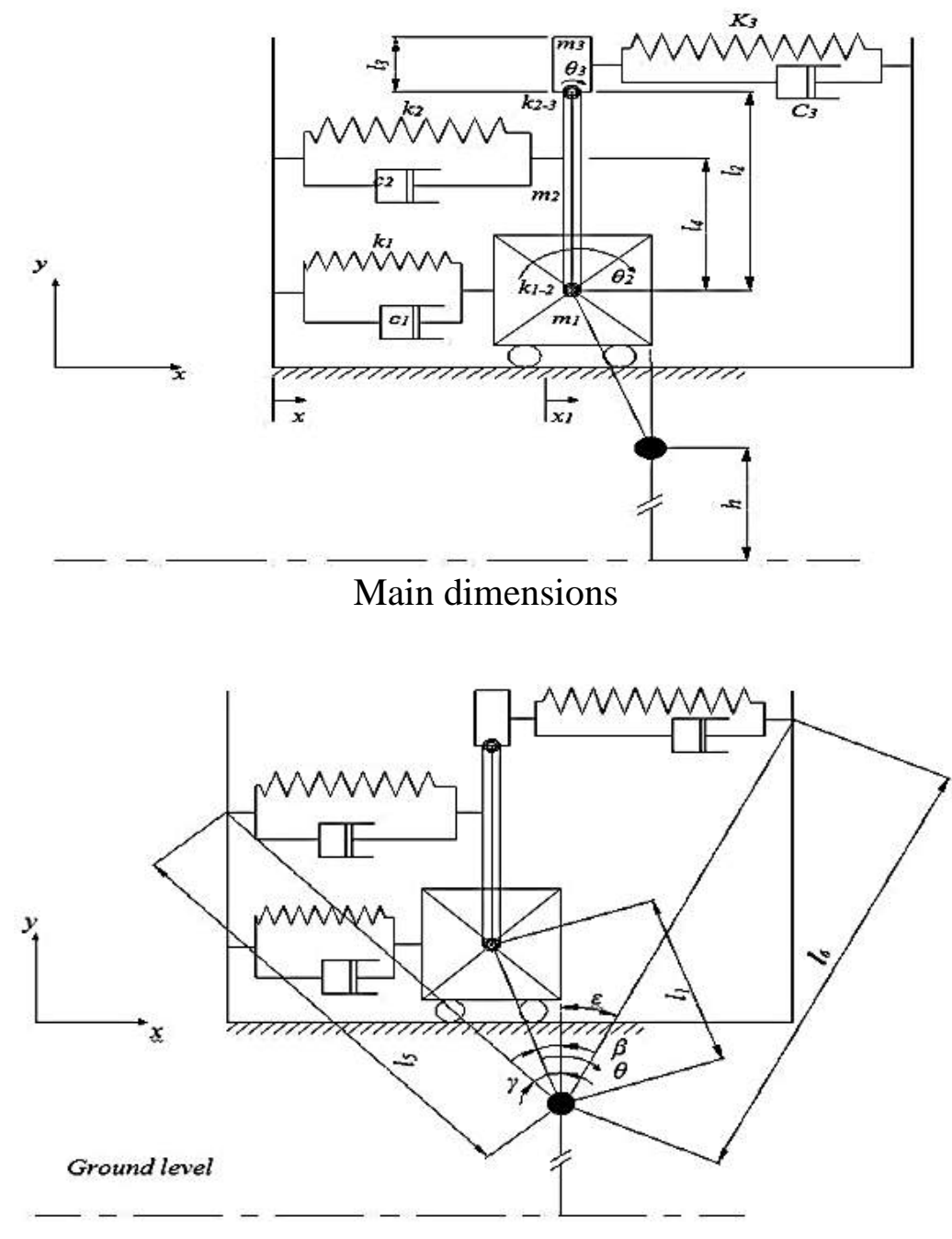

Other dimension

Figure 5. Multi-body occupant model. 

as follows:

The equation of motion of the human body, using Lagrange's method, is generated

$$
\frac{d}{d t}\left(\frac{\partial E}{\partial \dot{q}}\right)-\frac{\partial E}{\partial q}+\frac{\partial V}{\partial q}+\frac{\partial D}{\partial \dot{q}}=0
$$

where E, V and D are the kinetic energy, potential energy and the Rayleigh dissipation function of the system, respectively. q represents the variables; $\left(\mathrm{x}_{1}\right)$ longitudinal movement of the occupant's lower body $\left(\theta_{2}\right)$ the rotational angle of the occupant's middle body and $\left(\theta_{3}\right)$ the rotational angle of the occupant's upper body, respectively, while $\dot{q}_{1}$ is the variables' velocities.

The kinetic energy of the system can be written as:

$$
E=\frac{m_{1} \cdot v_{1}^{2}}{2}+\frac{m_{2} \cdot v_{2}^{2}}{2}+\frac{m_{3} \cdot v_{3}^{2}}{2}+\frac{I_{1}}{2} \cdot \dot{\theta}^{2}+\frac{I_{2}}{2} \cdot \dot{\theta}_{2}^{2}+\frac{I_{3}}{2} \cdot \dot{\theta}_{3}^{2}
$$

where $\mathrm{v}_{1}, \mathrm{v}_{2}$ and $\mathrm{v}_{3}$ are the equivalent velocities of the lower, middle and upper bodies of the occupant, respectively. $\mathrm{I}_{1}, \mathrm{I}_{2}$ and $\mathrm{I}_{3}$ are the rotational moment of inertia of the lower, middle and upper bodies about the $\mathrm{CG}$ of each body, respectively. The equivalent velocities of the three bodies of the occupant can be calculated as follows:

$$
v_{1}^{2}=\dot{X}_{m_{1}}^{2}+\dot{Y}_{m_{1}}^{2}
$$

where the displacement and velocity of the lower body in $\mathrm{x}$ direction can be calculated as:

$$
\begin{gathered}
X_{m_{1}}=x_{1}+l_{1 \circ} \cdot(\sin \beta-\sin (\beta-\theta) \\
l_{1}=\sqrt{\left[l_{1 \circ} \sin (\beta-\theta)-x_{1}\right]^{2}+\left[l_{1 \circ} \cos (\beta-\theta)\right]^{2}}
\end{gathered}
$$

based on the small change in $\theta$ during the crash event, $1_{1}$ has been taken as constant in all equations.

$$
\dot{X}_{m_{1}}=\dot{x}_{1}+l_{1} \cdot \dot{\theta} \cdot \cos (\beta-\theta)
$$

and the displacement and velocity of the lower in y direction can be calculated as:

$$
\begin{gathered}
Y_{m_{1}}=l_{1} \cdot(\cos (\beta-\theta)-\cos \beta) \\
\dot{Y}_{m_{1}}=l_{1} \cdot \dot{\theta} \cdot \sin (\beta-\theta)
\end{gathered}
$$

substituting Eqs.(13.d) and (13.f) in Eqs.(13.a), the equivalent velocity of the lower body can be written as:

$$
v_{1}^{2}=\dot{x}_{1}^{2}+l_{1}^{2} \cdot \dot{\theta}^{2}+2 \cdot \dot{x}_{1} \cdot l_{1} \cdot \dot{\theta} \cdot \cos (\beta-\theta)
$$


By repeating the previous steps of Eq. (13), the equivalent velocities of the middle and upper bodies and the total kinetic energy can be calculated.

where $\mathrm{X}_{\mathrm{mi}}$ is the resultant longitudinal displacement and $\mathrm{Y}_{\mathrm{mi}}$ is the resultant vertical displacement. (i: denotes body position 1: lower, 2: middle and 3: upper), $1_{1}, 1_{2}$ and $1_{3}$ are the distance from the vehicle's CG to the lower body's CG, middle body length and upper body length, respectively. It is assumed that $l_{1}$ is constant due to the insignificant change of its length during the crash. $\beta$ is the angle between the vertical centreline of the vehicle and the line between the vehicle's CG and the CG of the lower body (see Figure 6).

The potential energy of the system can be written as:

$$
\begin{gathered}
V=m_{1} \cdot g \cdot\left(h+z+Y_{m_{1}}\right)+m_{2} \cdot g \cdot\left(h+z+Y_{m_{1}}+\frac{l_{2}}{2} \cdot \cos \theta_{2}\right) \\
+m_{3} \cdot g \cdot\left(h+z+Y_{m_{1}}+l_{2} \cdot \cos \theta_{2}+\frac{l_{3}}{2} \cdot \cos \theta_{3}\right)+\frac{k_{1}}{2} \cdot\left(\delta_{1}-\delta_{s 1}\right)^{2} \\
+\frac{k_{2}}{2} \cdot\left(\delta_{2}-\delta_{s 2}\right)^{2}+\frac{k_{3}}{2} \cdot\left(\delta_{3}-\delta_{s 3}\right)^{2}+\frac{k_{R 12}}{2} \cdot\left(\theta_{2}-\theta\right)^{2}+\frac{k_{R 23}}{2} \cdot\left(\theta_{3}-\theta_{2}\right)^{2}
\end{gathered}
$$

where the deflection on the lower and upper seatbelt springs can be calculated as:

$$
\begin{gathered}
\delta_{1}=x_{1}-x \\
\delta_{2}=x_{1}-x+l_{4} \cdot \sin \theta_{2}-l_{5} \cdot(\sin \gamma-\sin (\gamma-\theta)) \\
\delta_{3}=x_{1}-x+l_{2} \cdot \sin \theta_{2}+\frac{l_{3}}{2} \cdot \sin \theta_{3}-l_{6} \cdot(\sin (\varepsilon+\theta)-\sin \varepsilon)
\end{gathered}
$$

where $h$ is the vehicle's CG height. $k_{1}, k_{2}, k_{R 12}$ and $k_{R 23}$ are the lower seatbelt stiffness, upper seatbelt stiffness, the spring stiffness of the pivot 1, and the spring stiffness of the pivot 2 , respectively. $\delta_{1}, \delta_{2}, \delta_{3}, \delta_{\mathrm{s} 1}, \delta_{\mathrm{s} 2}$ and $\delta_{\mathrm{s} 3}$ are the total deflection of the lower seatbelt spring, total deflection of the upper seatbelt spring, total deflection of the airbag, the initial slack length of the lower seatbelt spring, the initial slack length of the upper seatbelt spring, and the initial slack length of the airbag, respectively. $1_{4}$ is the distance between the pivot 1 and the contact point between the upper seatbelt spring and the middle body, $1_{5}$ is the distance between the vehicle's CG and the contact point between the upper seatbelt spring and the vehicle compartment, $1_{6}$ is the distance between the vehicle's CG and the steering wheel, $\gamma$ is the angle between the line $1_{5}$ and vertical centre line of the vehicle's $C G$, and $\varepsilon$ is the angle between the line $l_{6}$ and vertical centre line of the vehicle's CG.

The Rayleigh dissipation function can be written as:

$$
\begin{aligned}
D= & \frac{c_{1}}{2} \cdot\left(\dot{x}_{1}-\dot{x}\right)^{2}+\frac{c_{2}}{2} \cdot\left(\dot{x}_{1}-\dot{x}+l_{4} \cdot \dot{\theta}_{2} \cdot \cos \theta_{2}-l_{5} \cdot \dot{\theta} \cdot \cos (\gamma-\theta)\right)^{2} \\
& +\frac{c_{3}}{2} \cdot\left(\dot{x}_{1}-\dot{x}+l_{2} \cdot \dot{\theta}_{2} \cdot \cos \theta_{2}+\frac{l_{3}}{2} \cdot \dot{\theta}_{3} \cdot \cos \theta_{3}-l_{6} \cdot \dot{\theta} \cdot \cos (\varepsilon+\theta)\right)^{2}
\end{aligned}
$$

where $c_{1}, c_{2}$ and $c_{3}$ are the damping ratio of the lower seatbelt damper, the damping ratio of the upper seatbelt damper, and the damping ratio of the airbag damper, respectively.

The occupant model has been validated by the authors in previous work by comparing its results with the former finite element human model and crash test [21]. The 
validation test showed that the results from the mathematical model and the previous results are in good agreement.

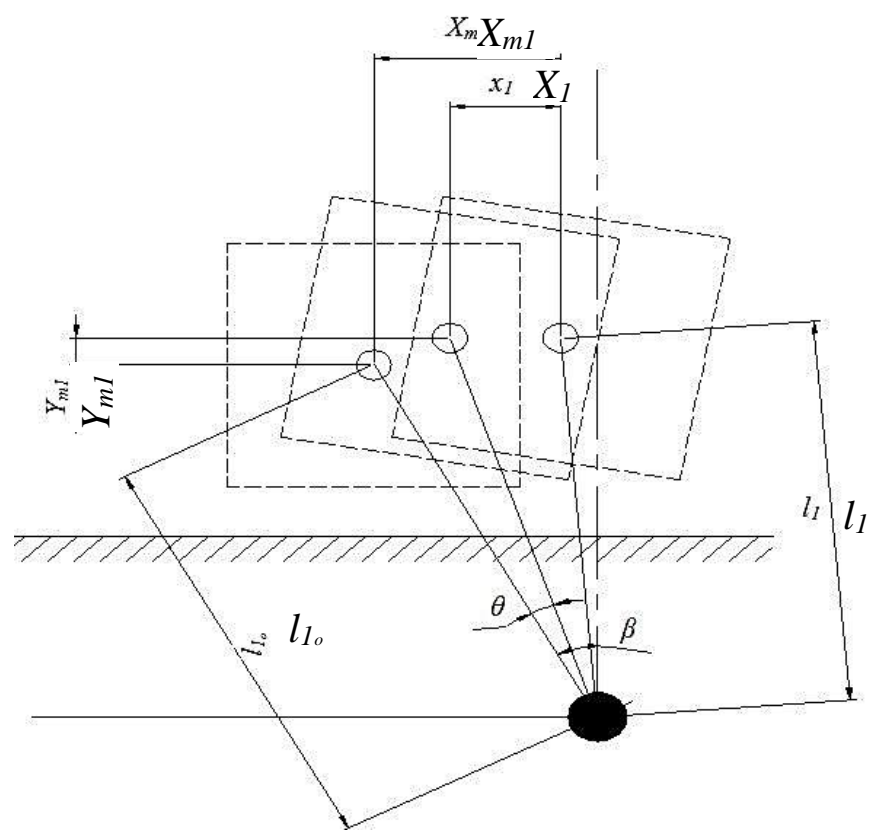

Figure 6. A schematic diagram of the occupant's lower body movement during the impact.

\section{RESULTS AND DISCUSSION}

Six different cases of vehicle crash scenarios are investigated in this paper. The first three cases are: free rolling, anti-lock braking system (ABS) and ABS integrated with underpitch control (UPC). The next three cases are the repetition of the first three cases using the extendable bumper system. These different cases of vehicle crash scenarios are described as follows:

Case 1: Free rolling - in this case, a vehicle collides with a barrier/vehicle without applying any type of control.

Case 2: ABS - in this case, the anti-lock braking system is applied before and during the collision.

Case 3: ABS + UPC - in this case, the UPC is developed with the aid of the ASC system using the fuzzy logic controller. The idea of the UPC controller is to give the vehicle body negative pitch angle before the crash and try to maintain the vehicle in this case until it collides with the barrier. The objective of the UPC system is to obtain the minimum pitching angle and acceleration of the vehicle body during the crash.

Cases 4, 5 and 6 are a repetition of cases 1,2 and 3 using the extendable bumper system, respectively.

\section{Primary Impact Results}

The ADAS detected that the crash is unavoidable at $1.5 \mathrm{sec}$ prior to the impact [22], the VDCS and the extendable bumper has been activated in this short time prior to the impact. The values of different parameters used in numerical simulations are given in Table 1 [23]; while the damping coefficient and the length of the hydraulic cylinder of the extendable bumper system are chosen to be $20000 \mathrm{~N} . \mathrm{s} / \mathrm{m}$, and $0.4 \mathrm{~m}$, respectively. The 
vehicle is adapted to collide with the barrier at $55 \mathrm{~km} / \mathrm{hr}$ speed. Prior to the collisions, the front-springs forces are equal to zero in the equations of motion. The front-end springs forces are re-deactivated at the end of the collision (vehicle velocity equal zero/negative values) and the behaviour of the vehicle in post-collision is captured. Front-end deformation, vehicle body acceleration, pitching angle and pitching acceleration are depicted to assess the crash behaviour of the vehicle.

Table 1. The values of the different parameters, which are used in the simulations.

\begin{tabular}{ccccccccc}
\hline Parameter & $\mathrm{m}$ & $\mathrm{I}_{\mathrm{yy}}$ & $\mathrm{k}_{\mathrm{Sf}}$ & $\mathrm{k}_{\mathrm{SrR}}$ & $\mathrm{c}_{\mathrm{fR}}=\mathrm{c}_{\mathrm{fL}}$ & $\mathrm{c}_{\mathrm{rR}}=\mathrm{c}_{\mathrm{rL}}$ & $\mathrm{l}_{\mathrm{f}}$ & $\mathrm{l}_{\mathrm{r}}$ \\
\hline \multirow{2}{*}{ Value } & 1200 & 1490 & 36.5 & 27.5 & 1100 & 900 & 1.185 & 1.58 \\
& $\mathrm{~kg}$ & $\mathrm{~kg} . \mathrm{m}^{2}$ & $\mathrm{kN} / \mathrm{m}$ & $\mathrm{kN} / \mathrm{m}$ & $\mathrm{N} . \mathrm{s} / \mathrm{m}$ & $\mathrm{N} . \mathrm{s} / \mathrm{m}$ & $\mathrm{m}$ & $\mathrm{m}$ \\
\hline
\end{tabular}

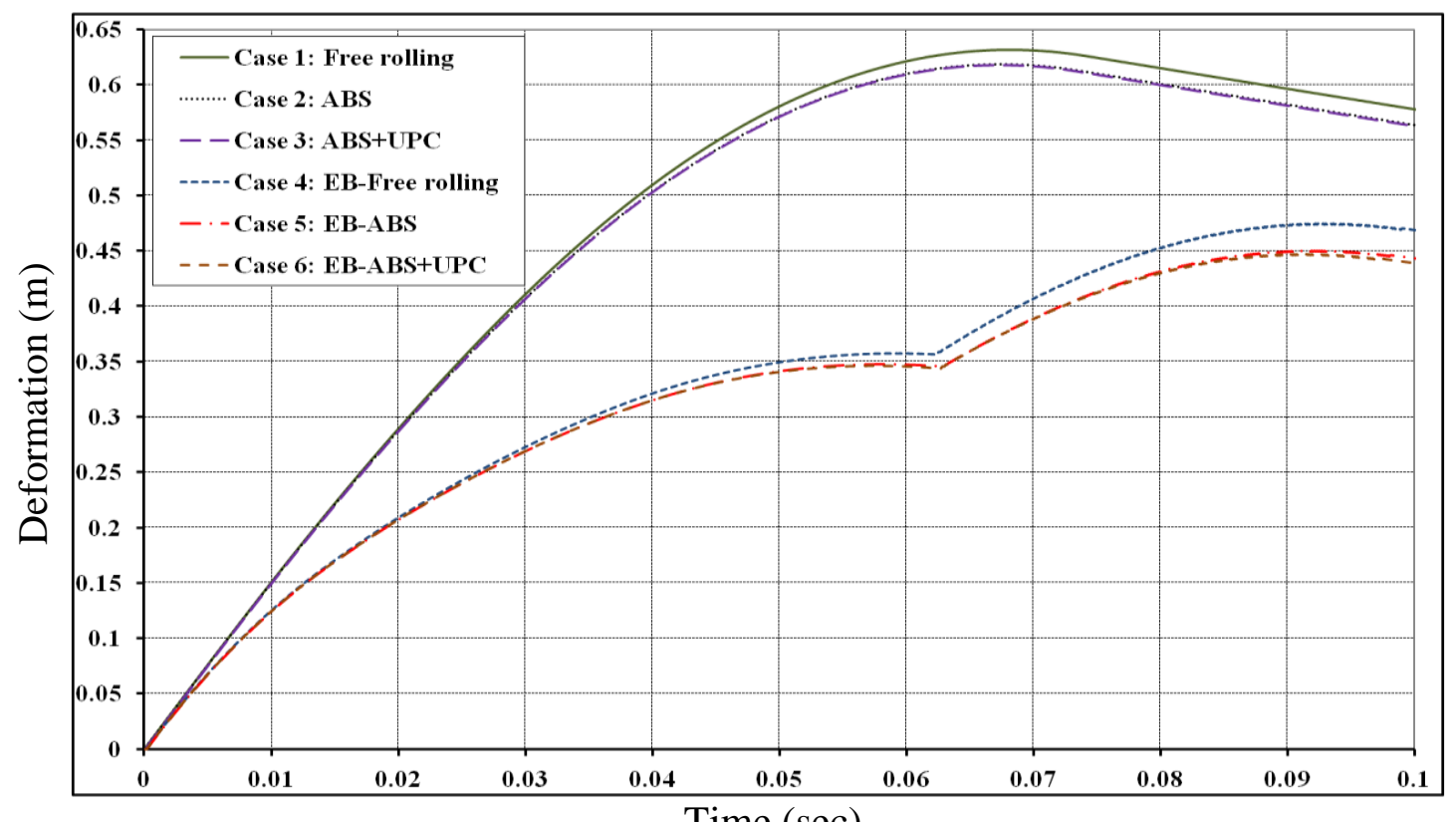

Time $(\mathrm{sec})$

Figure 7. Deformation of the front-end structure.

Figure 7 shows the front-end structure's deformation-time histories for all different VDCS cases. The deformation of the front-end is divided into two phases. The first is the compression phase, which is considered complete when the vehicle reaches the velocity of the obstacle (zero for the barrier). Next is the restitution (rebound) phase, which ends when the force of deformation results are equal to 0 . It is noticed that for the first set of results (without the extendable bumper), the deformation increased to reach its maximum value (different for each case) and then decreased slightly due to front-end springs rebound. Slight differences in the maximum deformation of the vehicle's front-end are found in each case; however, a reduction of about $20 \mathrm{~mm}$ of the maximum deformation is obtained in cases 2 and 3 compared to case 1 (free rolling). For the second set of results (with the extendable bumper), the deformation increased slowly to reach a specific point (around $0.06 \mathrm{sec}$ ); at this point, the extendable bumper is completely deformed. Then, it increased rapidly to reach its maximum value and then decreased slightly due to the rebound. 


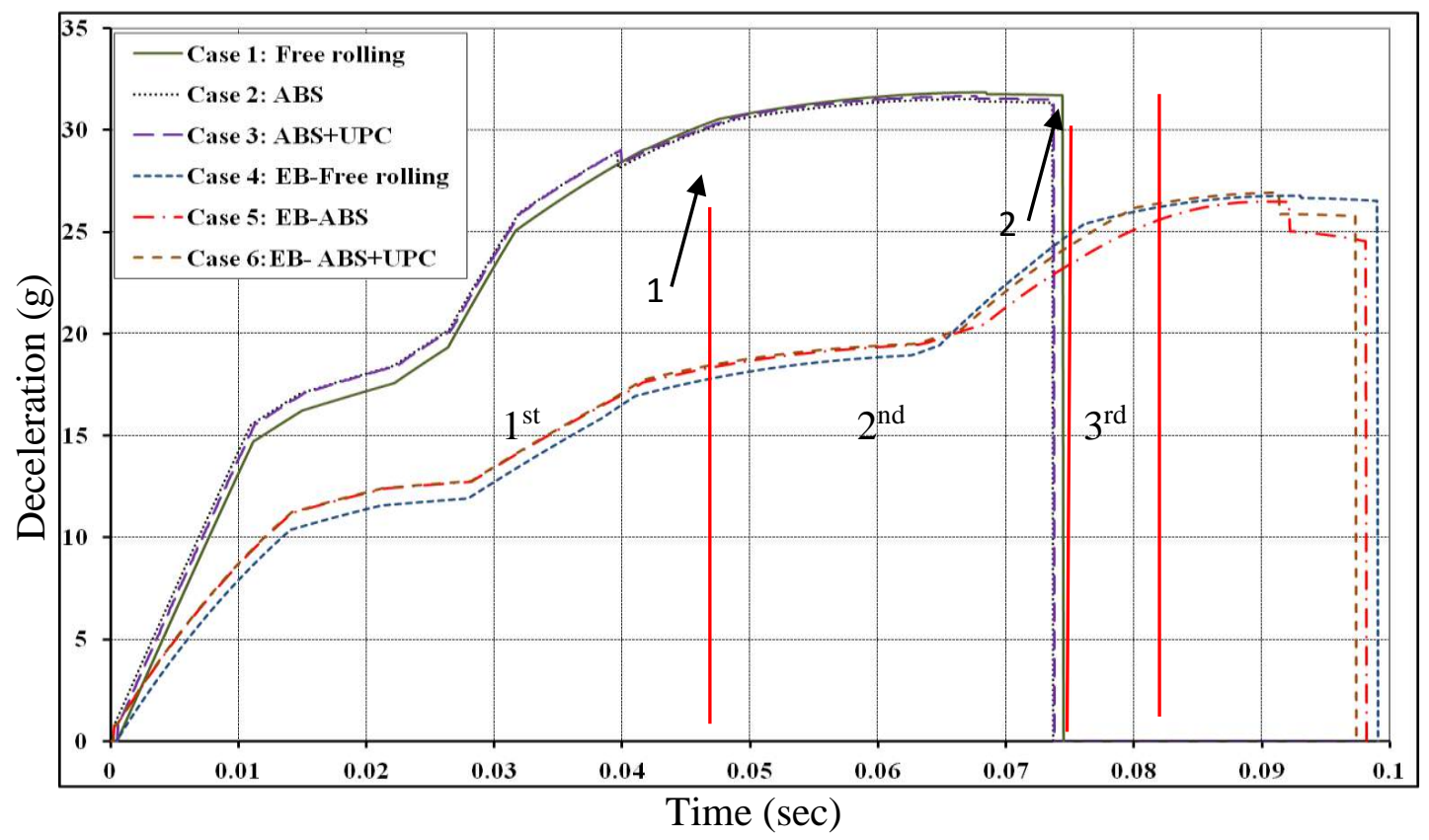

Figure 8. Vehicle body deceleration.

The deceleration - time histories of the vehicle body in all cases - are presented in Figure 8. For the first set of results, the deceleration-time history can be divided into three stages. The first stage represents the increase of the vehicle's deceleration before the front wheels reach the barrier. This deceleration is caused by the deformation of the front-end and the effect of braking forces [17]. In this stage, a slightly higher deceleration is noticed for cases 2 and 3 compared to the free rolling case. In the second stage, the front wheels reach the barrier and stop moving; therefore, their braking effects are vanished. The braking force is one source of vehicle deceleration, so when its effect is vanished at the beginning of this stage, a rapid reduction in the vehicle body deceleration occurs (arrow 1, Figure 8). At the end of this stage, the vehicle stops and starts moving in the opposite direction. In addition, the braking force changes its direction and another drop in the vehicle deceleration is noticed as shown in Figure 8 (arrow 2). The maximum deceleration is observed at this stage because the front-end structure is crushed and acts as a solid with a great increase in stiffness [24]. It is observed that the deceleration in this stage is almost the same for all crash cases. At the third stage, a condition of allowing the front-end springs to be rebounded for a very short time is applied during the simulation analysis. During this stage, the vehicle moves back and the deformation of the front-end decreases as shown in Figure 7. At the end of this stage, the non-linear front-end springs are deactivated and the vehicle's deceleration suddenly drops to a value of zero. This fast drop is due to the assumption of immediate stopping the effect front-end springs after a very short time of rebound [17]. For the second set of simulation, the front wheels do not reach the barrier; therefore, the second stage does not exist. From the point of impact until the extendable bumper is completely compressed (between 0.06 and $0.07 \mathrm{Sec}$ ), a slight higher deceleration is noticed for cases 5 and 6 compared to case 4 . After this point, a rapid deceleration of the vehicle body is noticed and the maximum deceleration is almost the same for all cases. It is clear from Figure 8 that the vehicle sustains lower deceleration $(27 \mathrm{~g})$ when the extendable bumper is used alongside VDCS compared to (32 g) when only the VDCS is applied. It is also obvious that the effect of VDCS on vehicle deceleration is insignificant. 
Figure 9 shows the vehicle's pitch angle-time histories in all cases. The VDCS is applied 1.5 second before the collision; therefore, the vehicle body impacts the barrier at different values of pitch angles according to each case as shown in Figure 9. The vehicle's pitch angle then reaches its maximum values (normally after the end of the crash) according to each case. Following this, the pitch angle reduced to reach negative values and then bounces to reach its steady-state condition. In general, when the vehicle is subjected to pitch motion, it shakes forth and back (positive and negative pitch angles) several times before reaching its stability again [25]. In the case of free rolling, the vehicle body pitching angle is generated solely due to the different impact forces between the upper and lower front-end springs, while in case 2 and 5 (only ABS is applied), an extra pitching moment is generated due to the braking force. This explains how the maximum pitch angle is observed in these cases. When the under pitch technique is applied along with ABS (cases 3 and 6), the vehicle is given a negative pitch angle prior to impact, and the UPC forces generate a negative pitch moment prior and during the impact. In these cases, a great improvement of the vehicle pitching is obtained.

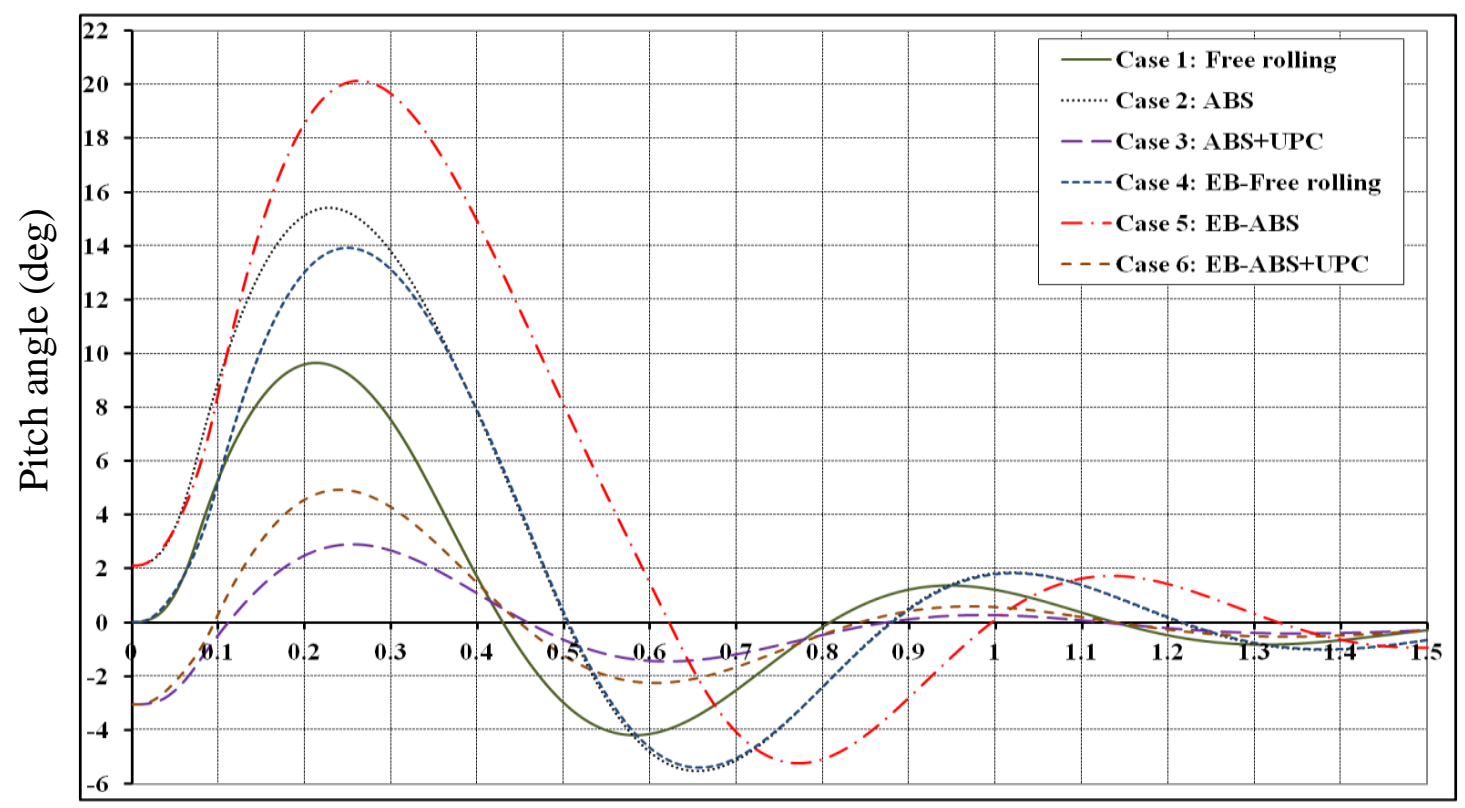

Time (sec)

Figure 9. Vehicle body pitch angle.

It is noticed that the vehicle pitching increases when the extendable bumper is used. This can be concluded by noting that the maximum pitching angle is 9.6, 15.4 and 2.9 degrees in cases 1, 2 and 3 respectively, compared to 14, 20, and 4.9 degrees for cases 4 , 5 and 6 , respectively. There are two factors that cause these higher values of pitching angles. Firstly, the crushing force is only applied on the lower rails through the extendable bumper at the early stage of collision, while the upper rails do not reach the barrier yet. Secondly, the braking force of the front wheels is continually activated during the crash period because the front wheels do not reach the barrier in this period. These two factors are the main source of generating pitching moment to the vehicle body.

The vehicle pitch acceleration-time histories are depicted in Figure 10 for all six cases. The pitch acceleration is increased very quickly at the early stage of the impact to reach its maximum value for each case due to the high pitching moment that is generated from the collision. This peak in the vehicle pitch acceleration during the impact is due to 
the main load path for the crash pulse being transmitted through the structure to the cabin below the vehicle centre of mass [17]. At the end of the collision, all pitching moments due to the crash are equal to zero, vehicle speeds are negative with very low values, and the vehicle pitch angles are still positive. This means that the vehicle is now controlled by the tyres and suspension forces, which have already generated moments in the opposite direction of the vehicle pitching. This describes the reason for the high drop and the changing direction from positive to negative on the vehicle pitch acceleration at the end of the crash [11].

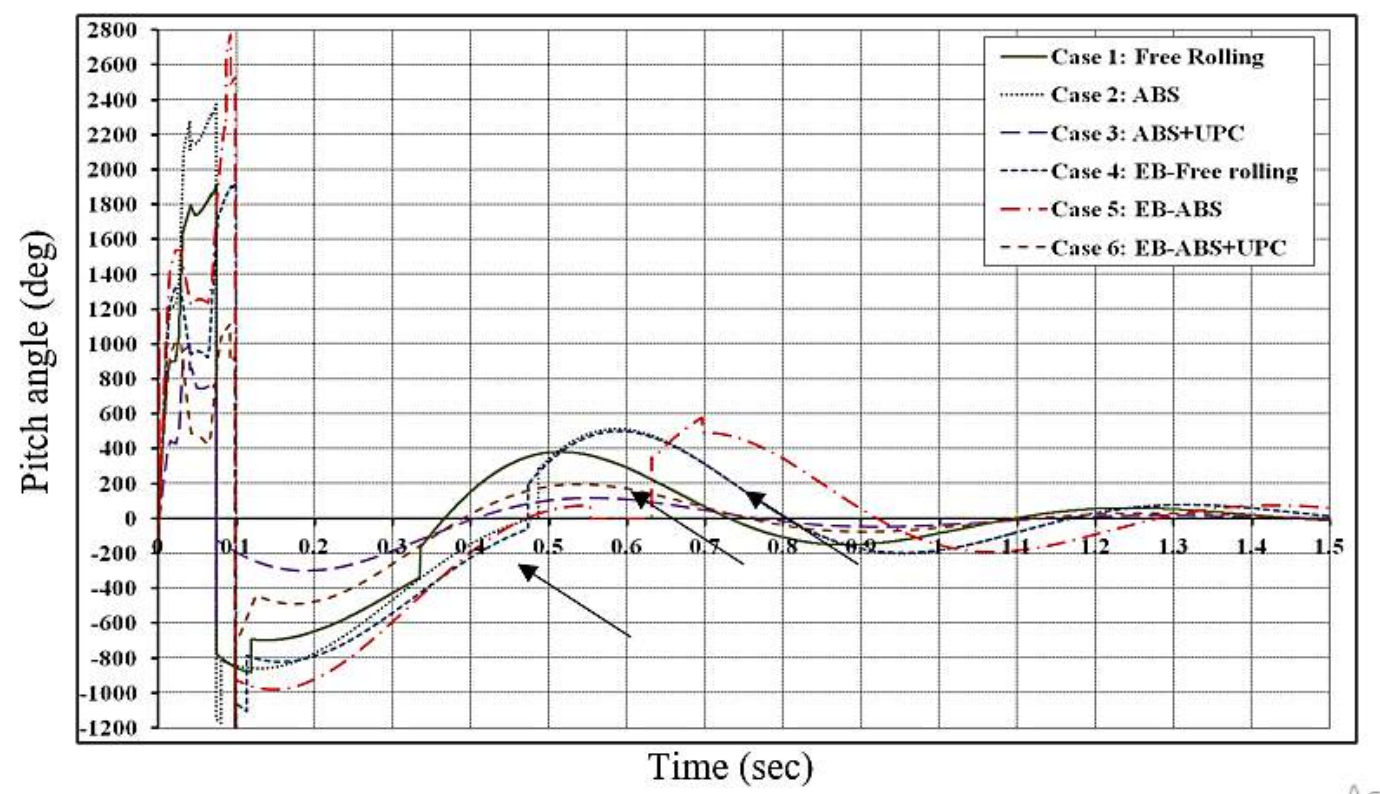

Figure 10. Vehicle body pitch acceleration.

As shown in Figure 10, the vehicle's maximum pitching acceleration occurs at the end of the collision and the greatest value of the maximum pitching acceleration is observed in case 5 (EB-ABS). The following highest value of pitching acceleration is noticed in case 2 (ABS). Meanwhile, the pitching angle in cases 1 and 3 is lower than the pitching angle in cases 4 and 6 , the maximum pitching acceleration is almost the same for cases 1 and 4 (free rolling and EB- free rolling); and 3 and 6 (ABS + UPC and EB- ABS + UPC), respectively. That means, although the extendable bumper does not help reduce the pitching angle, it does not cause higher pitching acceleration except in case 5 (EBABS). This is because when the extendable bumper is used, the crash force is applied only below the vehicle centre of mass, in addition to the braking force, which is the main source of pitching motion [25]. The lowest value of the vehicle pitching acceleration is detected in cases 3 and 6 at almost the same value. The reduction of the vehicle pitch acceleration in these cases is also noteworthy; it decreases from about $2400 \mathrm{deg} / \mathrm{s}^{2}$ in case of free rolling to about $1000 \mathrm{deg} / \mathrm{s} 2$ in case 3 and 6 . Because of the vehicle's rear wheels left the ground during the vehicle pitching, a sudden increase of the vehicle pitching acceleration is observed when the rear wheels re-contacted the ground (look at the arrows in Figure 10). This sudden increase in pitching acceleration does not exist in cases 3 and 6 because the rear wheels do not leave the ground in these cases.

\section{Secondary Impact Results}

The injury criteria in this paper have been taken as the occupant's pelvis deceleration, occupant's chest rotational acceleration, and head rotational acceleration. These injury 
criteria of the occupant have been determined based on the output data obtained from the vehicle dynamics/crash model. The vehicle output data (deceleration and pitching acceleration) due to the collision are transferred to the occupant as a sudden deceleration to all the body, and rotational movements of the head and chest. It is assumed that at initial condition, the occupant's chest and head are in a vertical position. When the VDCS is applied (1.5 Sec prior collision), the occupant's chest and head take different angles in this short time according to each case and then collide with the barrier with these different angles. It is also important to mention that the front airbag is activated at the point of impact. The occupant data used in the numerical simulation are presented in Table 2 [26], while the total stiffness of the two seatbelt springs is $98.1 \mathrm{kN} / \mathrm{m}$ with a damping coefficient of $20 \%$ [8], and then it is distributed between the upper and lower seatbelt springs by a ratio of 2:3, respectively [27]. Airbag's spring stiffness is $5 \mathrm{kN} / \mathrm{m}$ and the damping coefficient is $20 \%$. The slacks of the seatbelt springs are assumed zero, and the slack of the airbag is $0.05 \mathrm{~m}$.

Table 2. The values of the occupant parameters.

\begin{tabular}{cccccccc}
\hline Parameter & $\mathrm{m}_{1}$ & $\mathrm{~m}_{2}$ & $\mathrm{~m}_{3}$ & $\mathrm{k}_{\mathrm{R} 12}$ & $\mathrm{k}_{\mathrm{R} 23}$ & $\mathrm{~L}_{2}$ & $\mathrm{~L}_{3}$ \\
\hline \multirow{2}{*}{ Value } & 26.68 & 46.06 & 5.52 & 280 & 200 & 0.427 & 0.24 \\
& $\mathrm{~kg}$ & $\mathrm{~kg}$ & $\mathrm{~kg}$ & $\mathrm{Nm} / \mathrm{rad}$ & $\mathrm{Nm} / \mathrm{rad}$ & $\mathrm{m}$ & $\mathrm{m}$ \\
\hline
\end{tabular}

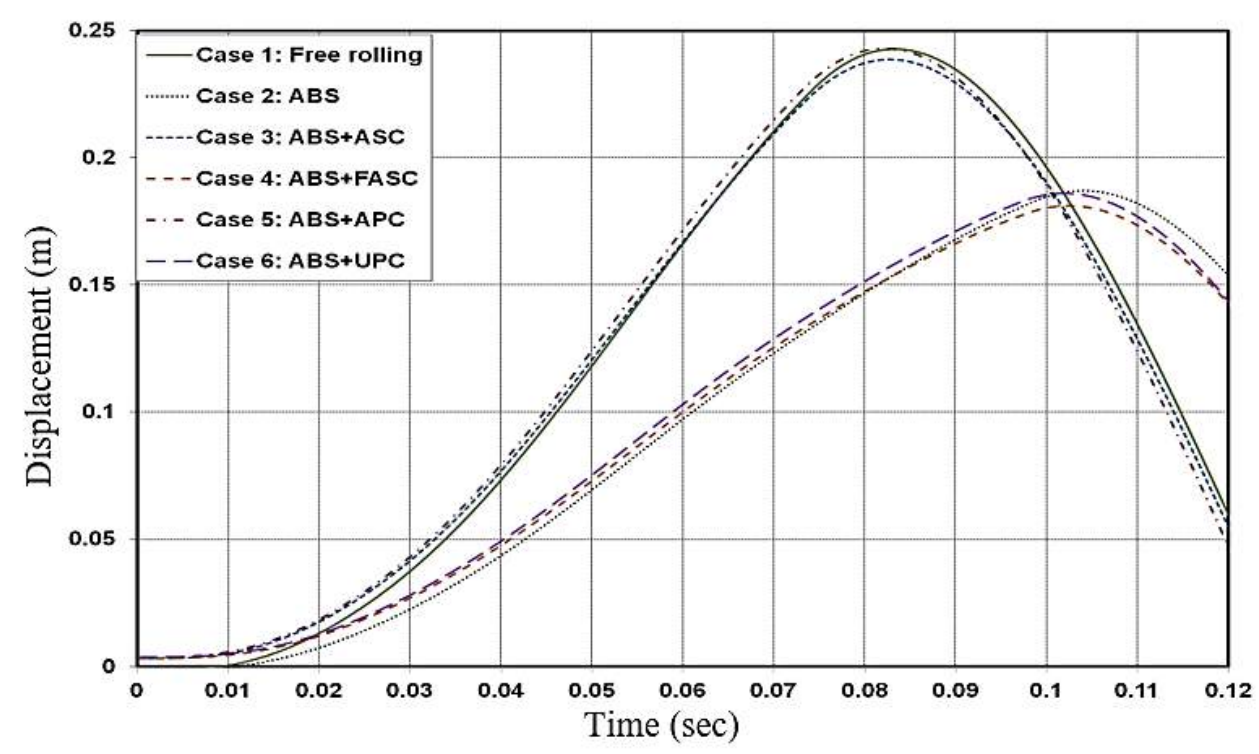

Figure 11. Occupant's pelvis displacement for all cases.

The longitudinal displacement of the pelvis is depicted for all cases in Figure 11; it increases forward to reach its maximum position almost at the end of impact, and then returns due to the seatbelt springs effect [28]. The fundamental advantage of the extendable bumper is to absorb more crash energy with the ability to use more distance available for crush. Therefore, the significant reduction in the pelvis' longitudinal displacement shown in Figure 11 is a logic. It is noticed that for the first set of results (without the extendable bumper), there are slight differences in the maximum displacement of the occupant's pelvis. For the second set of results (with the extendable bumper), the pelvis' displacement increased slowly compared to the first set of results to 
reach its maximum value and then decreased slightly due to the seatbelt rebound. It is observed from Figure 11 that there are also insignificant differences between the values of the maximum displacement of the occupant's pelvis. It means that the effect of VDCS on the occupant's longitudinal motion is negligible [20]. However, the reduction in that displacement is doubled when the extendable bumper is applied along with the VDCS.

Figure 12 shows the pelvis deceleration for all cases; it is shown that it increases during the collision to reach its maximum values at the end of impact and then reduces due to the seat belt effect [29]. The sudden decrease of the deceleration (arrow 1 in Figure 12) is due to the reversal of the effect of the braking force at the end of impact when the vehicle changes its direction and starts to move [20]. It is observed that the maximum deceleration is almost the same for all cases of the first and second sets of results with very small differences. These small differences mean that the VDCS do have an insignificant effect on the pelvis relative displacement and deceleration.

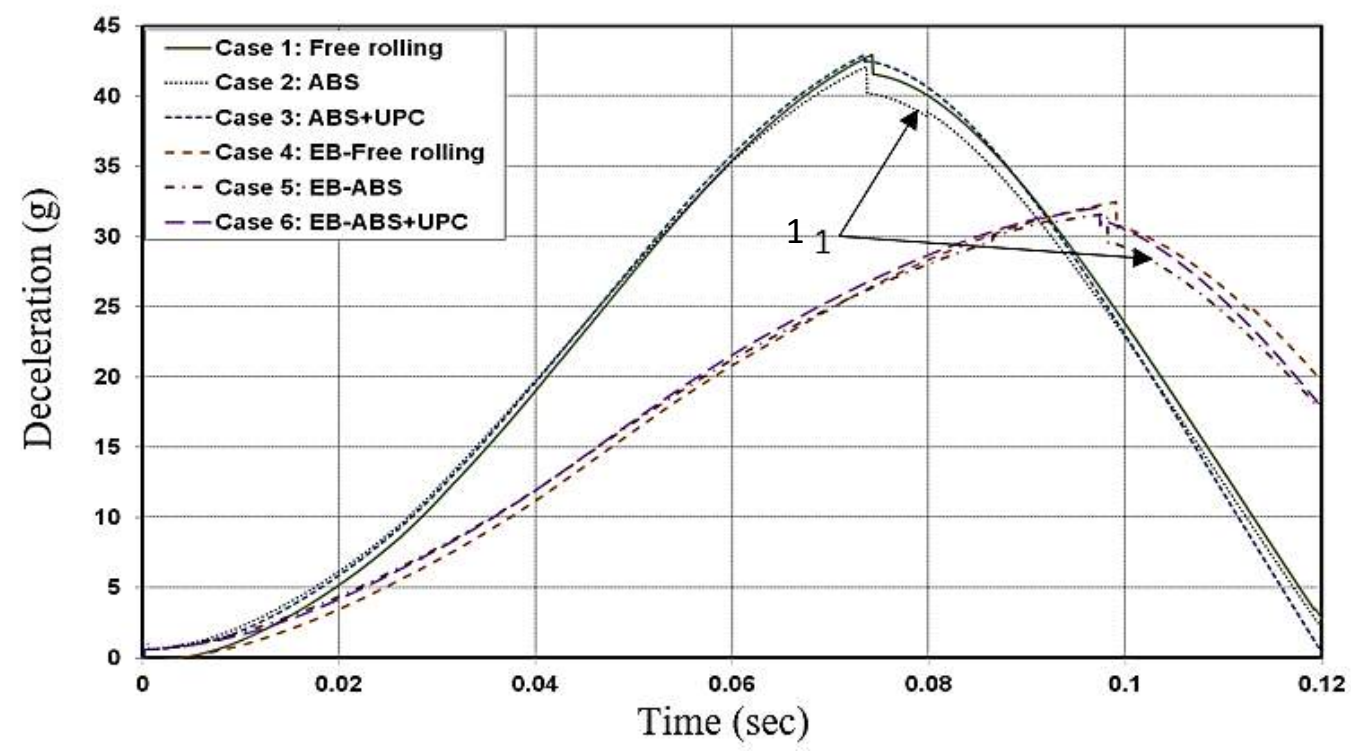

Figure 12. Occupant's pelvis deceleration for all cases.

The relative rotation angle and acceleration of the occupant's chest for all cases are shown in Figure 13 ( $a$ and b), respectively. The occupant's chest starts the collision with different rotational angles according to each case. The occupant takes this angle in the period of $1.5 \mathrm{sec}$ prior collision when the VDCS is applied. The chest rotational angle is increased to reach its maximum value after about 0.06 second from the end of impact. That is because at the end of impact, the occupant's chest has a positive rotational acceleration. The rotational motion reaches its maximum position when the rotational velocity is equal to zero. For the two sets of results, the maximum rotation angle is observed only when the ABS is applied, while the minimum one is observed when the control system sets to ABS + UPC case. For the first set of results (without the extendable bumper), the reduction of about 13 degrees is obtained while in the second set of results (with the extendable bumper), the reduction of about 15 degrees is obtained. 

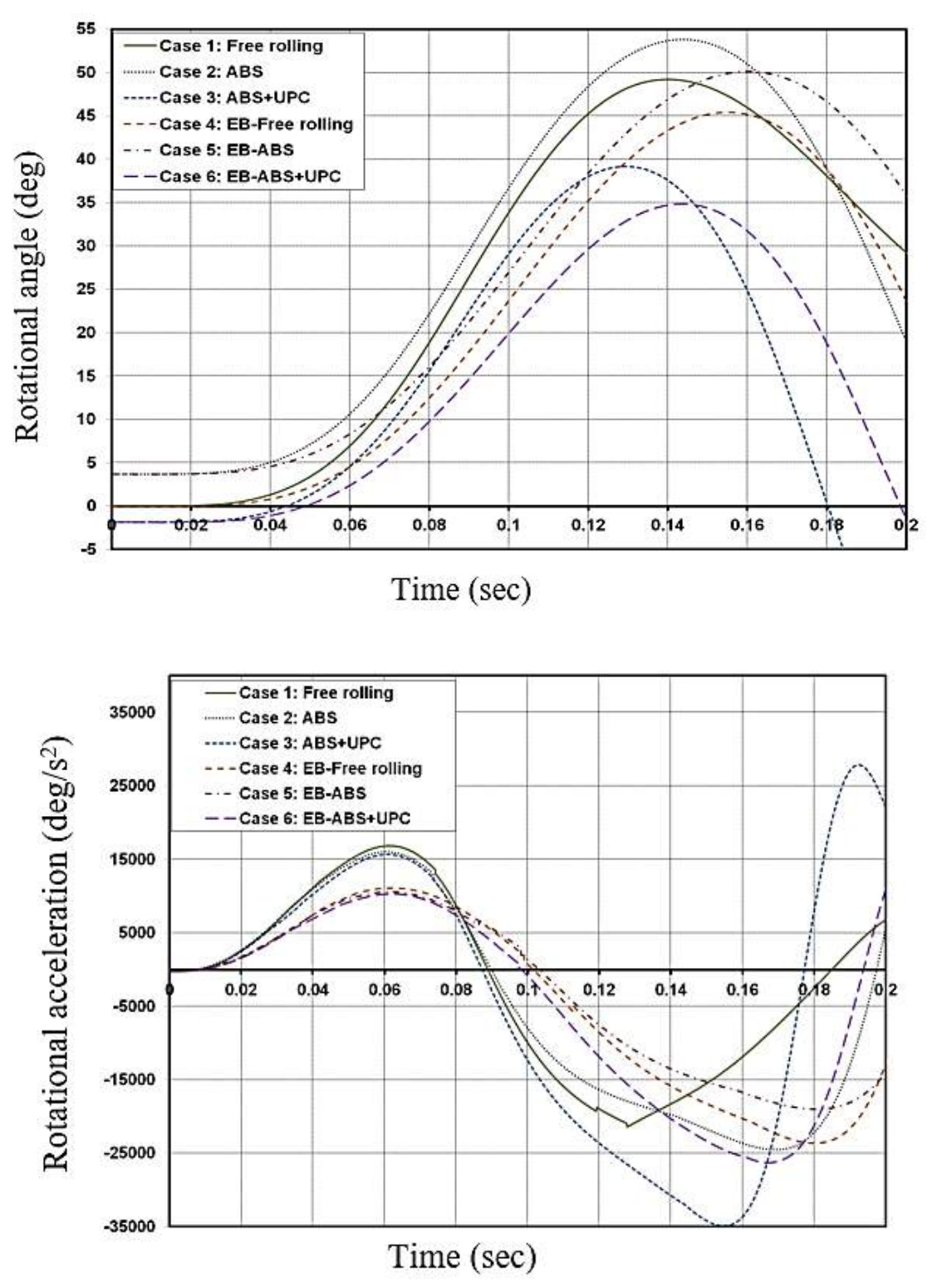

Figure 13. (a) Rotational angle of the occupant's chest about y axis for all cases; (b) Rotational acceleration of the occupant's chest for all cases.

In relation to the rotational acceleration, the positive rotational acceleration shown in Figure 13 (b) is due to the vehicle crash, while the negative maximum acceleration is due to the return of the seatbelt springs effect $[28,29]$. The chest rotational acceleration increases gradually to reach its maximum positive value and then reduces to reach its maximum negative value. For both sets of results, it is monitored that the minimum positive acceleration occurs when only the ABS is applied, while the minimum one in the negative acceleration happens in case 5 . The relative rotation angle between the occupant's chest and head is captured in Figure 14. The head rotation angle is increased to reach its first peak values, which occur during the increase of chest rotating. Then, it increased gradually in cases 1,2 and 5 to reach its second peak values or slightly decreased in cases 3, 4 and 6 due to the return of the occupant's chest. For the two sets of results, the peak value of the head rotational angle is observed when only the ABS is used, 
while the minimum one is detected when ABS + UPC system is used. The ABS + UPC system causes a reduction of about 20 degrees in the first set of results and about 15 degrees when the extendable bumper is used. Figure 15 shows the relative rotational acceleration of the occupant's head. For both sets of results, the maximum positive and negative acceleration are observed in free rolling case, while the minimum positive and negative values are seen when only the ABS is applied. As observed in the chest results, the head rotational angle and acceleration have started from different points related to the VDCS case.

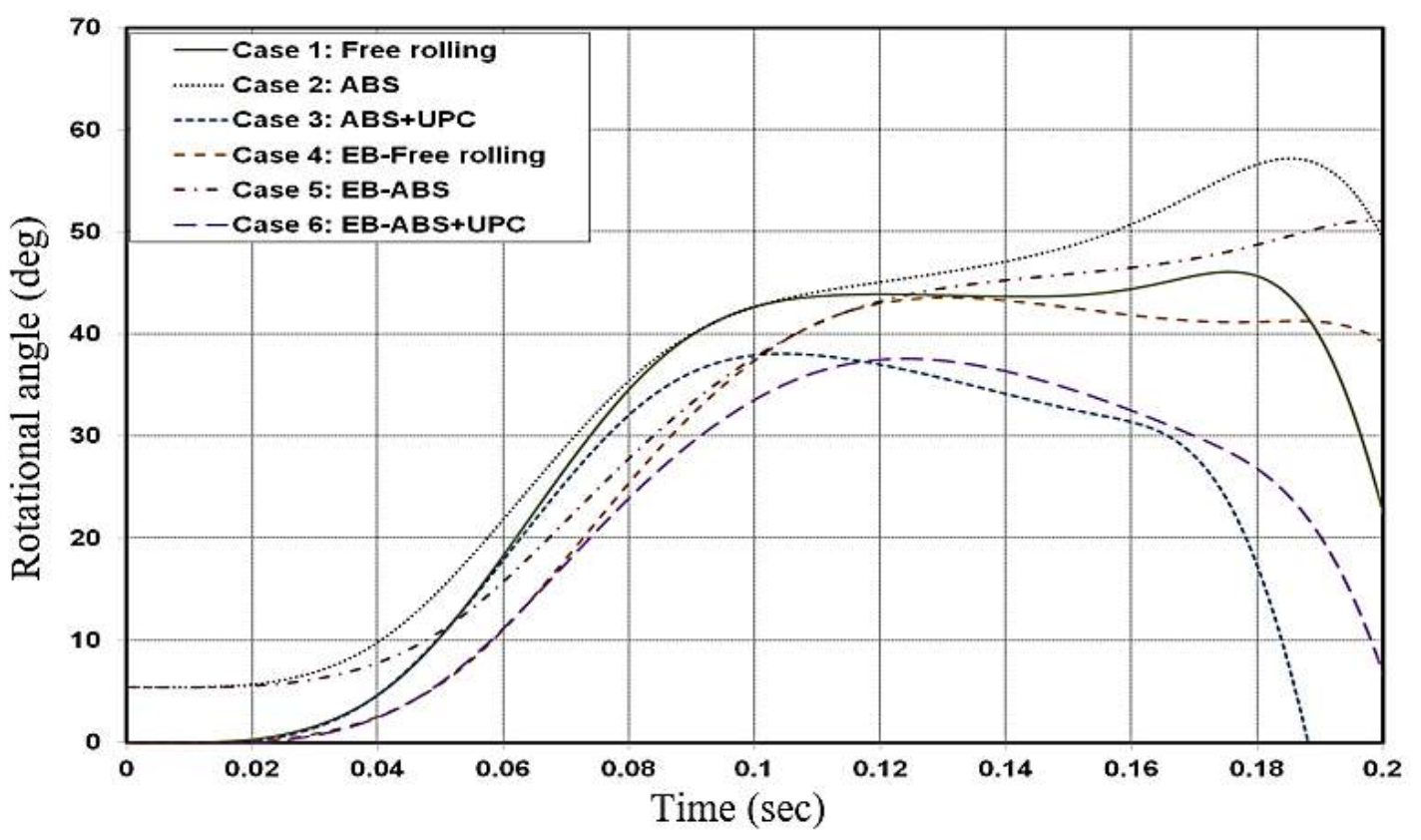

Figure 14. Rotational angle of the occupant's head about y axis for all cases.

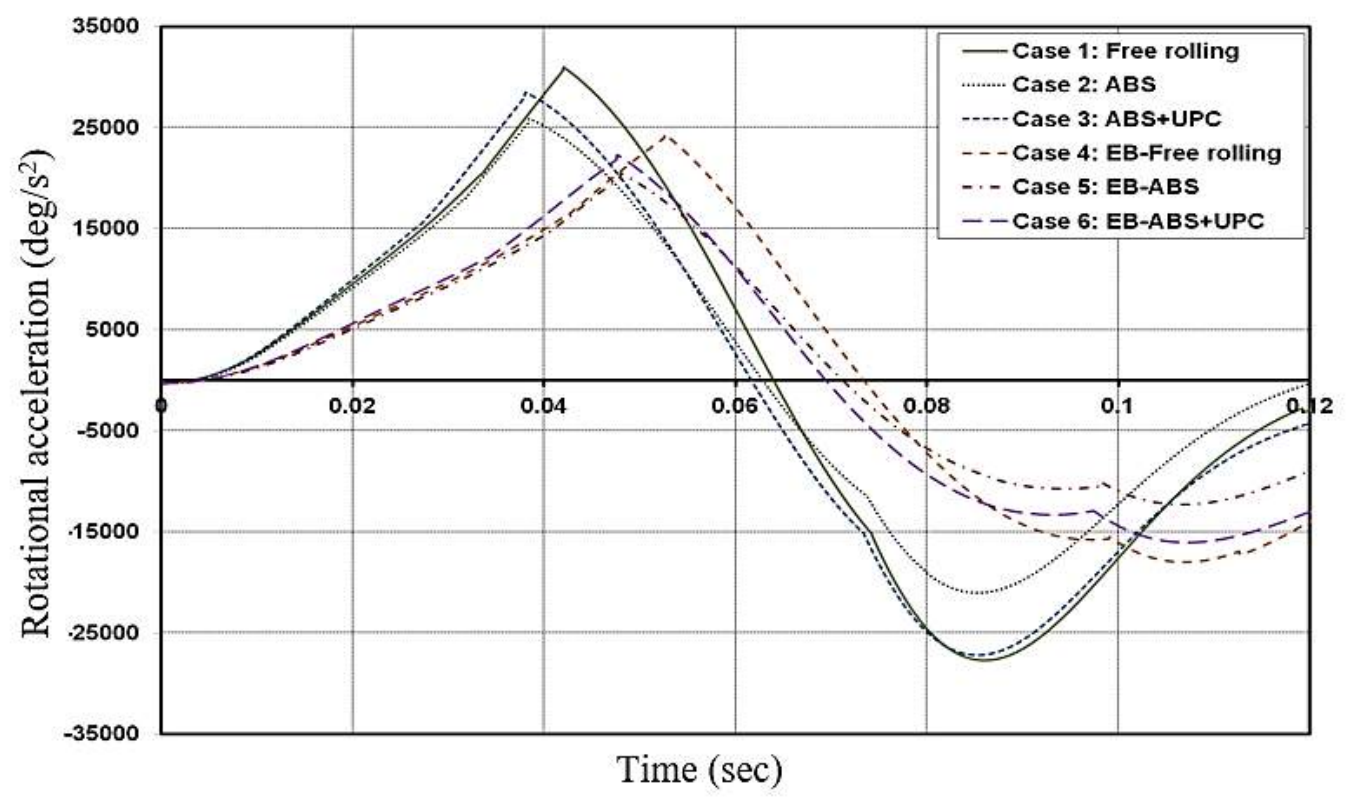

Figure 15. Rotational acceleration of the occupant's head about y axis for all cases. 
In relation to the occupant injury criteria, the occupant's head rotational accelerations appeared to be the major cause of strain-induced brain injury, which contributed to more than $80 \%$ of the brain strain and the peak amplitude of rotational acceleration must not exceed $9.4 \mathrm{krad} / \mathrm{s} 2(538.5 \mathrm{kdeg} / \mathrm{s} 2)$ [30]. The results show some improvement in the occupant injury criteria, which makes the crash event more survivable. Use of under pitch technique (cases 3 and 6) can help reduce the chest and head rotation angle, as well as head rotational acceleration. The VDCS affects the occupant's behaviour with different ways related to the applied case, and it can be seen that the application of frontal UPC alongside ABS (case 6) can be taken as the best case due to its great effect on the occupant's head (the most important part of the occupant's body).

\section{CONCLUSIONS}

A unique vehicle dynamics/crash mathematical model is developed to study the influences of VDCS integrated with the extendable bumper system on the vehicle collision mitigation. This model combines vehicle crash structures, vehicle dynamics control and extendable bumper systems. In addition, a multi-body occupant mathematical model has been developed to capture the occupant's dynamic response. It is shown from numerical simulations that the extendable bumper surpasses the traditional structure in absorbing crash energy at the same crash speed. Furthermore, it is shown that the extendable bumper brings significantly lower intrusions and helps keep the vehicle deceleration within desired limits. Although using the extendable bumper causes an increase in vehicle pitching angle, it does not affect the maximum pitching acceleration. The results obtained from different applied cases show that the VDCS affect the crash situation, by different ratios related to each case, positively. The deformation of the vehicle front-end structure is reduced when the VDCS is applied, and this reduction in the vehicle deformation is greater when the extendable bumper is used. The vehicle body deceleration is insignificantly changed within the applied cases of VDCS. The vehicle pitch angle and its acceleration are dramatically reduced when the ABS is applied alongside the UPC system. It is also shown that the extendable bumper beats the traditional structure in occupant injury criteria. The effect of the VDCS is quite minimal in terms of occupant relative displacement and deceleration. However, there is a significant effect on the rotations angle and acceleration of the occupant's chest and head.

\section{ACKNOWLEDGMENT}

The authors would like to thank the Egyptian Government and the Faculty of Engineering, Ain Shams University for supporting this research through the Phd scholarship funding (grant no. 2004-2/3/56). The authors also acknowledge with sadness, the contribution of Prof. Dave Crolla, who passed away during the period of this research.

\section{REFERENCES}

[1] Tamura M, Inoue H, Watanabe T, Maruko N. Research on a brake assist system with a preview function. SAE Technical Paper; 2001.

[2] Sugimoto Y, Sauer C. Effectiveness estimation method for advanced driver assistance system and its application to collision mitigation brake system. 
Proceedings of the 19th International Technical Conference on the Enhanced Safety of Vehicles. 2005. p. 5-148.

[3] Ghasemi A, Rouhi S. A safe stable directional vehicular platoon. Proceedings of the Institution of Mechanical Engineers, Part D: Journal of Automobile Engineering. 2014.

[4] Elmarakbi A, Zu J. Crashworthiness improvement of vehicle-to-rigid fixed barrier in full frontal impact using novel vehicle's front-end structures. International journal of automotive technology. 2005;6:491-9.

[5] Elmarakbi $\mathrm{A}, \mathrm{Zu} \mathrm{J}$. Crash analysis and modeling of two vehicles in frontal collisions using two types of smart front-end structures: an analytical approach using IHBM. International Journal of Crashworthiness. 2006;11:467-83.

[6] Elmarakbi A, Zu J. Mathematical modelling of a vehicle crash with emphasis on the dynamic response analysis of extendable cubic nonlinear dampers using the incremental harmonic balance method. Proceedings of the Institution of Mechanical Engineers, Part D: Journal of Automobile Engineering. 2007;221:143-56.

[7] Elmarakbi A, Zu J. Incremental harmonic balance method for analysis of standard/smart vehicles-to-rigid barrier frontal collision. International journal of vehicle safety. 2007;2:288-315.

[8] Elmarkbi A, Zu J. Dynamic modeling and analysis of vehicle smart structures for frontal collision improvement. International journal of automotive technology. 2004;5:247-55.

[9] Wang J, Browne AL. An extendable and retractable knee bolster. 18th ESV Conference United States. 2003.

[10] Wang J. An extendable and retractable bumper. 19 th ESV conference2005. p. 05-0144.

[11] Chang JM, Rahman M, Ali M, Tyan T, El-Bkaily M, Cheng J. Modeling and design for vehicle pitch and drop of body-on-frame vehicles. SAE Technical Paper; 2005.

[12] Yu F, Feng J-Z, Li J. A fuzzy logic controller design for vehicle ABS with a online optimized target wheel slip ratio. International Journal of Automotive Technology. 2002;3:165-70.

[13] Ba T, Guan X, Zhang J. Vehicle predictive control based on the recursive subspace identification method. Proceedings of the Institution of Mechanical Engineers, Part D: Journal of Automobile Engineering. 2014: 0954407014555878.

[14] Alleyne A, Hedrick JK. Nonlinear adaptive control of active suspensions. Control Systems Technology, IEEE Transactions on. 1995;3:94-101.

[15] Yue C, Butsuen T, Hedrick J. Alternative control laws for automotive active suspensions. American Control Conference, 1988: IEEE; 1988. p. 2373-8.

[16] Mastandrea M, Vangi D. Influence of braking force in low-speed vehicle collisions. Proceedings of the Institution of Mechanical Engineers, Part D: Journal of Automobile Engineering. 2005;219:151-64.

[17] Hogan I, Manning W. The use of vehicle dynamic control systems for automotive collision mitigation. Automotive Electronics, 2007 3rd Institution of Engineering and Technology Conference on: IET; 2007. p. 1-10.

[18] Ahmad F, Mazlan S, Zamzuri H, Jamaluddin H, Hudha K, Short M. Modelling and validation of the vehicle longitudinal model. International Journal of Automotive and Mechanical Engineering. 2014;10:2042-56. 
[19] Elkady M, Elmarakbi A, MacIntyre J. The influence of a vehicle dynamics control system on the occupant's dynamic response during a vehicle collision. Proceedings of the Institution of Mechanical Engineers, Part D: Journal of Automobile Engineering. 2012:0954407012445698.

[20] Elkady M, Elmarakbi A, Crolla D. Simulation of a multibody occupant model during vehicle collision with different applications of vehicle control systems. Proceedings of the Institution of Mechanical Engineers, Part K: Journal of Multibody Dynamics. 2012:1464419312449576.

[21] Elkady M. Enhancement of vehicle crash and occupant safety: a new integrated vehicle dynamics control systems/front-end structure mathematical model: University of Sunderland; 2012.

[22] Jansson J, Johansson J, Gustafsson F. Decision making for collision avoidance systems. SAE Technical Paper; 2002.

[23] Alleyne A. Improved vehicle performance using combined suspension and braking forces. Vehicle System Dynamics. 1997;27:235-65.

[24] Brell E. Simplified Models of Vehicle Impact for Injury Mitigation. 2005.

[25] Ori T, Gbaha P, Asseu O, Le Bot A. Vehicle stopping distance by means of suspensions control. Asian J Sci Res. 2011;4:28-41.

[26] Ilie S, Tabacu S. Study of the occupant's kinematics during the frontal impact. Ann Oradea Univ Fascicle Manag Technol Eng VI (XVI). 2007:542-51.

[27] Paulitz T, Blackketter D, Rink K. Constant force restraints for frontal collisions. Proceedings of the Institution of Mechanical Engineers, Part D: Journal of automobile engineering. 2006;220:1177-89.

[28] Weaver J. A simple occupant dynamics model. Journal of biomechanics. 1968;1:185-91.

[29] Woo D, Choi S-B, Choi YT, Wereley NM. Frontal crash mitigation using MR impact damper for controllable bumper. Journal of Intelligent Material Systems and Structures. 2007;18:1211-5.

[30] Zhang J, Yoganandan N, Pintar FA, Gennarelli TA. Brain strains in vehicle impact tests.Annual Proceedings/Association for the Advancement of Automotive Medicine: Association for the Advancement of Automotive Medicine; 2006. p. 17. 